\title{
Access Toolkit for Education
}

Mike Wald, E.A. Draffan, Russell Newman, Sebastian Skuse, Chris Phethean

\author{
ECS, University of Southampton, UK
}

M.Wald@soton.ac.uk

\begin{abstract}
This paper describes three tools that have been developed to help overcome accessibility, usability and productivity issues identified by disabled students. The Web2Access website allows users to test any Web 2.0 site or software application against a series of checks linked to the WCAG 2.0 and other guidelines. The Access Tools accessible menu helps with navigation to portable pen drive applications that can assist with accessibility, productivity and leisure activities when on the move. The accessible Toolbar provides support for the majority of browsers and accessible websites through magnification, spellchecking, text to speech readout, dictionary definitions and referencing modification of text, page style, colour and layout.
\end{abstract}

Keywords: accessibility, tool, learning

\section{Introduction}

The Access Technologies Team ${ }^{1}$ at the University of Southampton School of Electronics and Computer Science (ECS) has developed a series of tools funded by the University and JISC TechDis ${ }^{2}$ to help overcome accessibility, usability and productivity issues identified by disabled students. One of the points that arose out of the JISC funded LexDis project ${ }^{3}$ [1] was the degree to which students were able to use their assistive technologies with Web 2.0 type services such as Facebook, blogs and wikis. It was found that those who did not need access tools, such as screen readers or keyboard only access, did not necessarily use their text to speech or spell checking software in these situations. There were also many students who did not have these technologies but still wanted to check their spelling and to change the look of the web pages they were reading. There were also those who wanted to access the web when using computers other than their own and they needed some form of support. One further issue that also arose was the general inaccessibility of some of the Web 2.0 sites. It was therefore decided that a more comprehensive approach was needed which is described further in this paper. Working through the issues discussed, a chart of document types was produced (table 1) to enable the team to make the pen drive application choices and add to the JISC TechDis Accessibility Essentials Guides ${ }^{4}$, which

1 http://access.ecs.soton.ac.uk

2 http://www.jisctechdis.ac.uk

3 http://www. lexdis.org

4 http://Www.jisctechdis.ac.uk/accessibilityessentials 
cover the creation of accessible Word, Adobe PDF and PowerPoint documents for online use.

Table 1. Chart of document types

\begin{tabular}{|l|l|}
\hline \multicolumn{2}{|c|}{ Document Type } \\
\hline Adobe PDF & Mind Mapping \\
\hline Animation/video/simulation & Presentation \\
\hline Audio & Project Management \\
\hline Computer Aided Design & Qualitative data analysis \\
\hline Database & References \\
\hline Desktop Publishing & Spreadsheet \\
\hline eBook/eText & Statistics \\
\hline Graphics & Web pages \\
\hline Maths/Science & Word processor \\
\hline
\end{tabular}

The tools have been beta tested by ECS MSc students as part of their coursework and assessment for a module on assistive technologies and universal design.

\section{Web2Access}

Web2Access allows users to test any Web 2.0 site or software application against a series of checks ${ }^{5}$ linked to the WCAG $2.0^{6}$ and other guidelines ${ }^{7} 89$.

5 http://WwW. web2access.org.uk/test

6 http://Www.w3.org/TR/WCAG20/

7 http://WwW. universaldesign.ie/useandapply/ict/itaccessib ilityguidelines/applicationsoftware/guidelines/

8http://Www.03.ibm.com/able/guidelines/software/accesssof tware.html

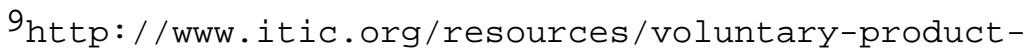
accessibility-template-vpat/\#1194.21 


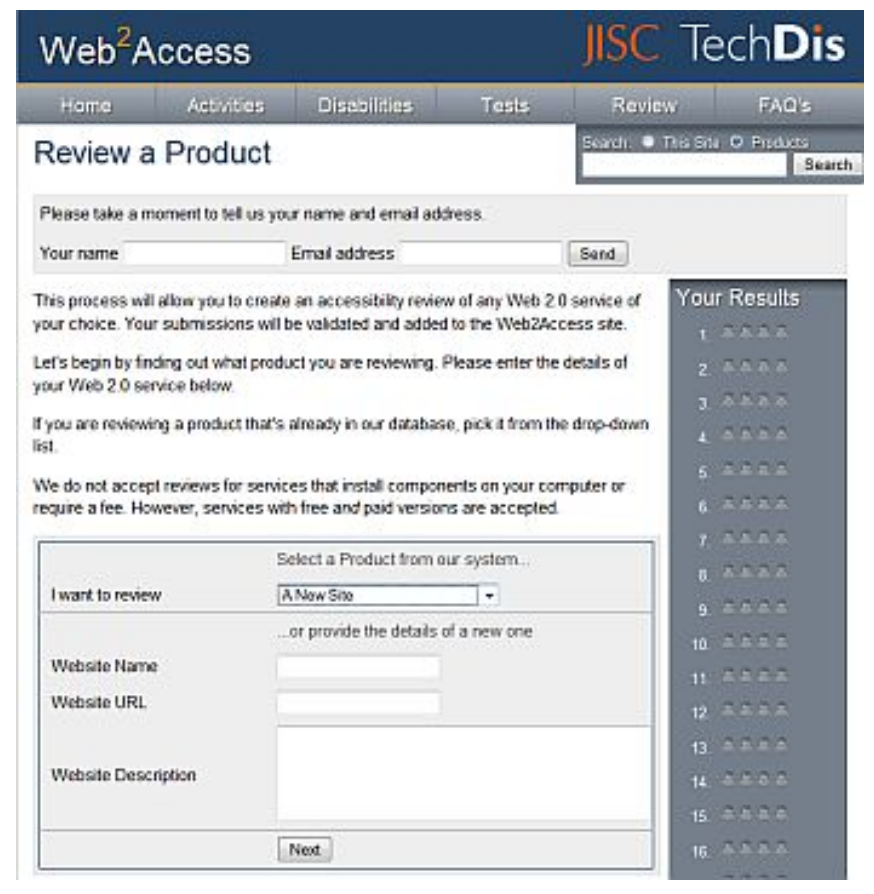

Fig. 1. Web2Access Interface reviewers screen

A summary of these is given in table 2 and table 3 . Results can be viewed for each site (see Fig. 2.) or for a particular disability (see Fig. 3). Over 150 Web 2.0 services have already been checked that will hopefully help users make suitable choices whether for personal use or for a teaching and learning environment. A wizard offers a step by step walk through with links to the techniques used for others to independently conduct tests (see Fig. 1.). Web2Access allows students, teachers and course developers to check the accessibility and usability of websites and applications they will be expected or required to use during their studies.

Table 2. Web 2.0 Service Tests

Login, Signup and Other Forms Accessible : registering with a service or site, then returning to sign-in and work with forms.

Image ALT Attributes: A text alternative enables a screen reader user to hear about the image Link Target Definitions: Link text needs to be understandable without a surrounding sentence or button.

Frame Titles and Layout: So screen reader users know where they are in the page

Removal of Stylesheet: to check how a site looks without style sheets

Audio/Video Features: text transcripts, captioning, and sign language can be helpful for deafness

Video/animations - audio descriptions: for scenes with no descriptive dialogue is essential.

Appropriate use of Tables: The order of content within the table and the use of row and column headers is important.

Tab Orderings Correct and Logical: in the same way that a page would linearise without any 
structural elements

Page Functionality with Keyboard

Accessibility of Text Editors: when using a keyboard or screen reader.

Appropriate Feedback with Forms: with no time restrictions and clear methods for pausing items or returning to a page to correct an error.

Contrast and Colour Check: Symbols and other items should not be provided in various colours without there also being other obvious differences to help those with colour deficiencies (colour blindness).

Page Integrity when Zooming: Zooming can improve readability but there are times when it also affects the layout of websites.

Text size, style, blinking elements and Readability: blinking at a certain rate can cause seizures and text needs to be understandable for all users

Table 3. Application Tests

Built in accessibility checks: using built-in system assistive technologies or accessibility options

Application works with External Assistive Technologies: additional screen reader, alternative input device and magnification should be able to work with all elements

Text or other alternatives for image elements.: It is important to ensure that colour alone is not used and text it easy to read.

Keyboard / Alternative input with focus: Fields, dialog boxes and focal points should all be reached in as logical a manner as possible so that the screen reader, keyboard and switch user can reach all the features in the application.

Labels for objects, fields or controls : Labels are linked to controls, objects, icons, and images so that screen reader users are aware of these elements within the application.

Audio alerts have visual cues: plus ability to use sounds sentries or show sounds. Audio options have volume control.

Alternatives for Video / Animation: such as transcripts or captions for those with hearing impairments. For visual impairments where there are long scenes with no descriptive dialogue. Media events offer user control: such as video or animation playing, audio and blinking /flashing notification events with no part of the screen flashing between $2 \mathrm{~Hz}$ and $55 / 60 \mathrm{~Hz}$.

Textual Information for screen reader: so user can hear when the action and changes are made within an application or when a dialog box appears on the screen.

Keyboard shortcut keys offered: where a program has its own access keys or shortcuts they must be clearly stated.

Save user preferences for style and zoom: For those with visual impairment and visual stress it may be important to be able to adapt the view of the application and content.

Timed events can be altered: so the user can work at their own speed.

Change colours and contrast: to support those who find the default settings unhelpful.

Uniform and standardised presentation: Consistency of navigation and task procedures is important.

Documentation: All instructions and help files should be clear, easy to read and available in accessible electronic format. 


\section{Results for $\underline{\text { VoiceThread }}$}

Web 2.0 Service Test Average: $44 \%$ Updated 10 August 2009

\section{Description}

Users upload images which become simple slideshows called Threads. Users can comment on Threads' by recording their voice andior doodling on the image. When the comment is played back, the doodle is drawn as it was by the user, showing the creation. Users can also comment with text and video.

\section{Accessibility Testing Results}

This site is mainly made using Flash and keyboard access has been compromised. The player is only usable with a keyboard in certain browsers such as Internet Explorer. It is impossible to change the text or contrast levels and user control is minimal, although the text size is good and feedback when uploading files is good. This service offers scope for adding audio descriptions and captions but its format means that using it with a screen reader is impossible.

\begin{tabular}{|c|c|c|c|}
\hline \# & Test & Score & Summary \\
\hline 1 & $\begin{array}{l}\text { Login, Signup and Other } \\
\text { Forms Accessible }\end{array}$ & 888 & $\begin{array}{l}\text { All forms are within a Flash component, and therefore cannot } \\
\text { be accessed with a keyboard. }\end{array}$ \\
\hline 2 & Image ALT Attributes & 888 & $\begin{array}{l}\text { The site is built using Flash, which cannot offer text alternatives } \\
\text { for images. }\end{array}$ \\
\hline 3 & Link Target Definitions & 888 & $\begin{array}{l}\text { Every button is within the Flash component, which cannot } \\
\text { provide titles or meaning for a screenreader. }\end{array}$ \\
\hline 4 & Frame Titles and Layout & 888 & $\begin{array}{l}\text { No frames or iframes, which can be disorientating for screen- } \\
\text { readers, are used in the design. }\end{array}$ \\
\hline 5 & Removal of Stylesheet & 888 & $\begin{array}{l}\text { The page is still understandable and functional when it is } \\
\text { viewed as a linear document, with no styles. }\end{array}$ \\
\hline 6 & AudioNideo Features & 888 & $\begin{array}{l}\text { It is a shame that the interface is not keyboard or screenreader } \\
\text { accessible as it is possible to join in with the conversation in } \\
\text { many different ways. }\end{array}$ \\
\hline
\end{tabular}

Fig. 2. Web2Access site results screen

\section{Access Tools}

There are many portable pen drive applications that can help with accessibility, productivity and leisure activities when on the move ${ }^{10}$ but these can be rendered virtually useless without an accessible pen drive menu . An accessible menu (see Fig. 4.) has therefore been developed to help with navigation to these applications. The settings allow for colour and font changes, large text and keyboard access. This pen drive has been developed for staff to check the accessibility and usability of websites and applications but should also be useful for students. A series of simple guides has also been made available that complement the JISC TechDis Accessibility Essentials. The tools that have been used to test the Web 2.0 services and applications have been added to the Access Tools download page along with a page of instructions.

10 http://access.ecs. soton.ac.uk/projects/access-tools 


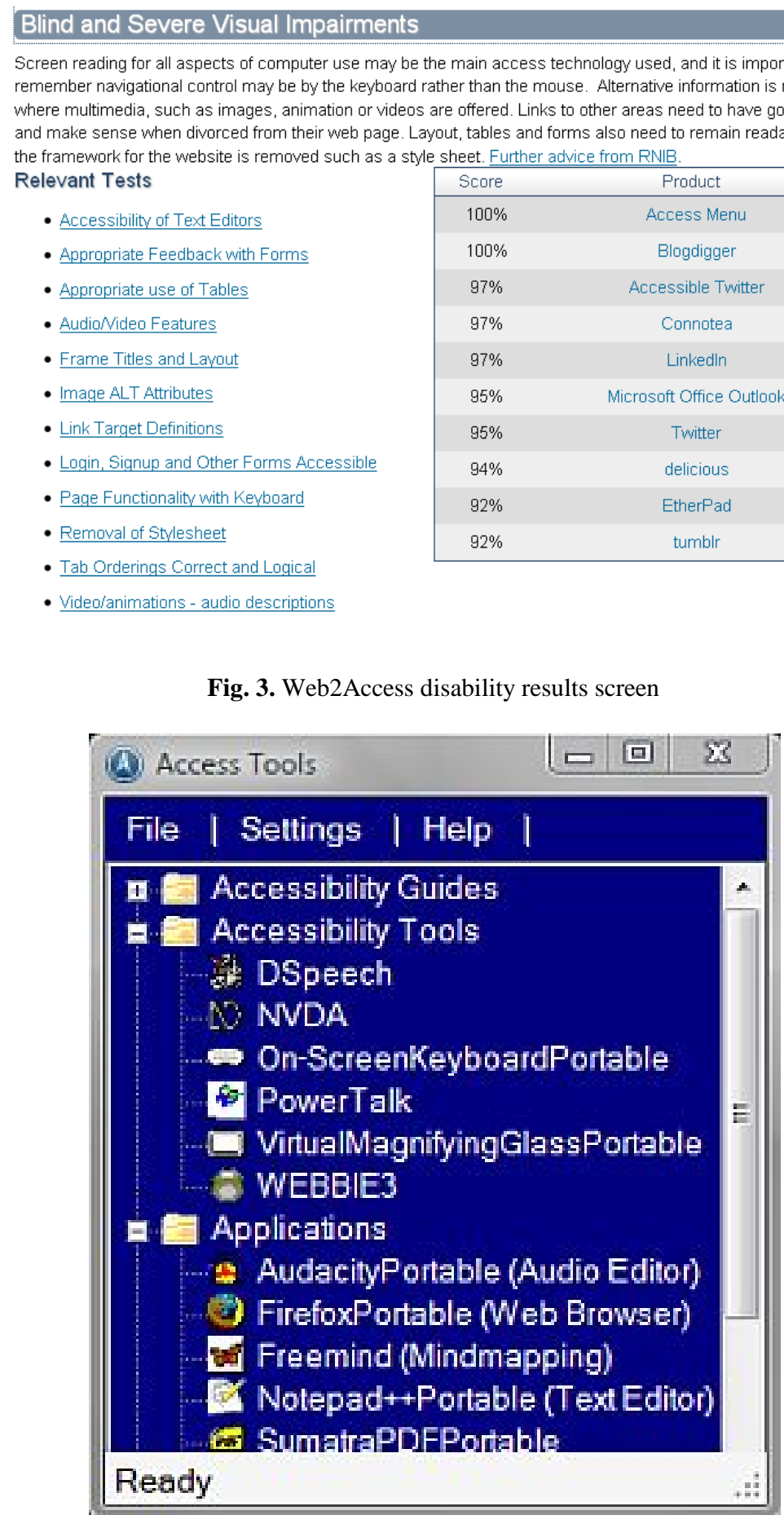

Fig. 4. Access Tools 


\section{Web 'ToolBar'}

Although most browsers offer ways to change the look and feel of websites whether through colour or text changes and the use of add-ons such as text to speech, these only work within that particular browser. It was felt that it would be useful to have a 'toolbar' that would work with all browsers and with the majority of accessible websites. ATBar ${ }^{11}$ (see Fig. 5.) can be simply run from your favourites/bookmarks and allows for text resizing, colour changes and text to speech. It can also be used with forms to check spelling and meanings of words. There is a tool for grabbing a web page's information such as the author, date and URL which may be handy when capturing sites for referencing and many other ideas have come up in discussion with those testing the toolbar. A button also allows users to report inaccessible websites to Fix the Web ${ }^{12}$ whose volunteers take these issues forward with website owners. The Toolbar is available under the BSD Open Source license. Users are free to copy, redistribute and study it as long as they leave the BSD license including original attribution intact. Modifications and derived works can be distributed under the terms of the BSD. An Arabic version of the toolbar funded by MADA centre ${ }^{13}$ and ATkit, a toolkit with which people can build their own version of ATbar customising it with the accessibility features that they want are currently under development and will be able to be demonstrated at ICCHP 2012.
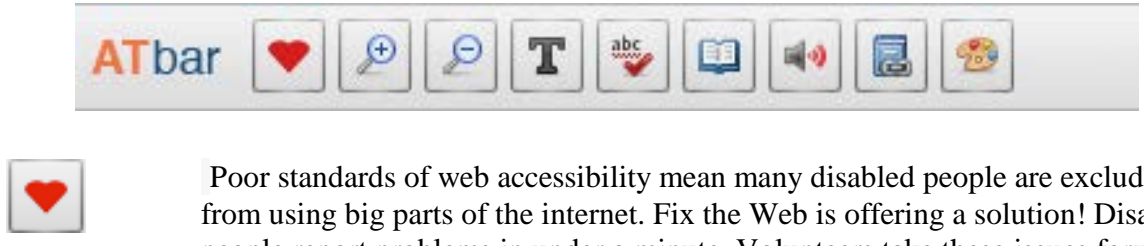

Poor standards of web accessibility mean many disabled people are excluded from using big parts of the internet. Fix the Web is offering a solution! Disabled people report problems in under a minute. Volunteers take these issues forward with website owners.

(4) Select the Magnifier buttons to increase or decrease the size of text. If text resizing makes the site unreadable you may wish to use your browser zoom feature to increase both the image and the text sizes.

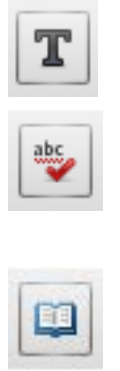

Select the Font button to choose a different type of font for the text and increase line spacing.

Select the Spell Checker button while writing in a plain text form. Suggestions are shown below the text-area after a pause. Disable the checker by selecting the button once more. Note that this may not work with rich-text editors that already have spell checkers.

Select the Dictionary button after highlighting a word in the web page and a definition from Wiktionary will appear.

11 http://access.ecs. soton.ac .uk/ToolBar /

12 http://www. fixtheweb. net/

13 http://mada.org.qa/ 
Select the text-to-speech button and choose whether you want the whole page read out loud, or just the highlighted text. You can highlight a portion of text before selecting the Text-to-Speech button to have only that text read out. Wait for that section to finish before highlighting a new section of text. Note that large pages, such as Facebook pages, may require you to select smaller portions of text at a time.

Select the References button and JISC TechDis Toolbar will scan the page for referencing information - it will collect as much as it can linked to authorship, dates, title of the page, name of the website and the URL. Once you have selected the green tick you can highlight the text and copy it into any notepad or word processor.

Select the Styles button to alter the colour of the JISC TechDis Toolbar, change the web page colours - background, text and links or choose pre-made page styles. Some web page colour changes also impact on the toolbar.

Select help to gain access to instructions for use.

Select the return menu if you wish to restore the webpage to its original style.

Select close to exit JISC TechDis Toolbar.

Fig. 5. ToolBar with explanation of icons and functions

\section{Conclusion}

This paper has described three tools that have been developed to help overcome accessibility, usability and productivity issues identified by disabled students. The Web2Access website allows users to test any Web 2.0 site or software application against a series of checks linked to the WCAG 2.0 and other guidelines. The Access Tools accessible menu helps with navigation to portable pen drive applications that can help with accessibility, productivity and leisure activities when on the move. The accessible toolbar provides for the majority of browsers and accessible websites support through magnification, text and page style, colour and layout modification, spellchecking, text to speech readout, dictionary definitions and referencing. The tools have been beta tested by MSc students as part of their course and assessment for a module on assistive technologies and universal design.

\section{References}

1. Wald, M., Draffan, E. A. and Seale, J. (2009) Disabled Learners' Experiences of Elearning. Jl. of Educational Multimedia and Hypermedia, 18 (3). pp. 341-361. 